\title{
Ovarian torsion in a postmenopausal patient of advanced age
}

Nur Gozde Kulhan, Mehmet Kulhan, Nahit Ata, Umit Arslan Nayki, Cenk Nayki, Pasa Ulug, Nesrin Yilmaz

Department of Obstetrics and Gynecology, School of Medicine, Erzincan University, Erzincan, Turkey

Submitted: 18 September 2017

Accepted: 9 November 2017

Arch Med Sci Civil Dis 2017; 2: e174-e176

DOI: https://doi.org/10.5114/amscd.2017.72549

Copyright (c) 2017 Termedia \& Banach

Ovarian torsion (OT) is one of the most common gynecological emergencies and can affect women of all ages [1]. Ovarian torsion has a bimodal age distribution, with a tendency to occur in young women (15-30 years old) and postmenopausal women. Moreover, the risk of OT increases in postmenopausal women with ovarian masses [2]. The mechanism of torsion of ovaries is unclear. Ovarian tumors, ovarian cysts and ovulation induction, which expands the ovarian volume, can be regarded as predisposing factors for OT [3]. As the size of the mass increases, the risk of torsion increases, until the mass becomes large enough to be fixed in place in the pelvis. In addition, masses that are fixed in place due to adhesions (e.g. endometrioma, tubo-ovarian abscess) or malignancy appear to be less likely to torse. It should be noted that OT rarely occurs in normal adnexa [4].

A 83-year-old postmenopausal woman presented with sudden onset of lower abdominal pain and discomfort. Her obstetric and gynecological history were unremarkable, without history of tubal sterilization. Hypertension and heart failure were recorded. On physical examination, she was afebrile but hypertensive. A tense mass in the right adnexa was identified in her vaginal examination and clinical examination of the abdomen revealed abdominal rebound and defense. Ultrasound revealed a well-defined, echo-free cystic mass $8 \mathrm{~cm} \times 10 \mathrm{~cm}$ in size on the right side of her uterus with a partial septations (Figure 1). No abnormal findings were demonstrated involving her left adnexa, and her uterus was normal. There was no ascites in the pouch of Douglas. Stroma was heterogeneous in appearance and locally hypoechoic and hyperechoic areas were noted in Doppler USG. Ovarian artery and venous blood were detected and the vascular signal was not completely lost. Her blood count and erythrocyte sedimentation rate were normal. Serum markers of ovarian malignancy were obtained and found to be within normal limits. After approximately $6 \mathrm{~h}$, the patient was taken to the laparotomy for a possible malignancy. The pre-operative diagnosis was torsion of malignant right ovarian cystic mass. An urgent laparotomy was performed which revealed a dark-red, round-shaped ruptured cystic lesion that twisted at the right infundibulo-pelvic ligament site in the right adnexa area. Her uterus and left ovary were normal and there were signs of necrosis on her right ovary and fallopian tube after reduction of the torsion (Figure 2). A right salpingo-oophorectomy was performed and it was sent for a histopathological examination. Frozen inspection was done. No pathologic

\author{
Corresponding author: \\ Mehmet Kulhan \\ Department of Obstetrics \\ and Gynecology \\ School of Medicine \\ Erzincan University \\ Basbaglar mah \\ 24030 Erzincan, Turkey \\ Phone: +90 5054918090 \\ E-mail: \\ Mehmet_kulhan@yahoo.com
}


diagnosis was performed owing to the complete necrosis of the surgical specimen, but there was no evidence of neoplastic proliferation (Figure 3). She was discharged after 3 days without any complications. No special complaint was noted during 6-month follow-up.

The incidence of OT is unknown [5]. Although the prevalent of OT is higher after the start of ovulation in adults, it may also be observed in postmenopausal women and adolescents. Ovarian torsion is a gynecological emergency encountered in all age groups. Ovarian torsion appears to occur infrequently in postmenopausal women. As an example, in a series of 301 postmenopausal women with ovarian neoplasms, 19 (6\%) had ovarian torsion [6]. When OT occurs, the ovary typically rotates around both the infundibulopelvic ligament and the utero-ovarian ligament. Rotation of the infundibulopelvic ligament causes compression of the ovarian vessels. Ovarian ischemia then occurs and can result in ovarian necrosis, infarction, and local hemorrhage.

The most common symptom is pain located in the lower abdominal region or pelvis that may radiate to the flank or thigh. Sudden onset of cramping pain or intermittent pain is possible. Temperature, white blood cell count, and erythrocyte sedimentation rate may be normal or slightly elevated.

A definitive diagnosis of OT is made by direct visualization of a rotated ovary at the time of surgical evaluation. Ultrasound is regarded as the imaging modality of choice for the diagnosis of OT. On the other hand, ultrasound alone often leads to misdiagnosis; therefore, it is important to detect normal blood flow by Doppler ultrasound. Even if abnormal blood flow is not confirmed, we cannot exclude the diagnosis of OT [7]. Although computed tomography (CT) scan findings are non-specific in OT, this modality may be helpful in ruling out this condition. The definite diagnosis of adnexal torsion can be established via surgery, laparoscopy or laparotomy, which can play both diagnostic and therapeutic roles [8].

There is no risk factor for postmenopausal OT except the right utero-ovarian ligament is longer physiologically than the left and ovarian mass. The right ovary cystic mass was seen as a risk factor in the present case.

For most premenopausal patients with OT, detorsion and ovarian conservation is recommended rather than salpingo-oophorectomy. Ovarian cystectomy is often performed if a benign mass is present. Cystectomy significantly reduces the risk of re-torsion by $50-75 \%$, compared to de-torsion. In recurrent cases, ovariopexy is preferred in order to reduce the risk of recurrence [9]. Patients with an ovarian mass that is suspicious for malignancy

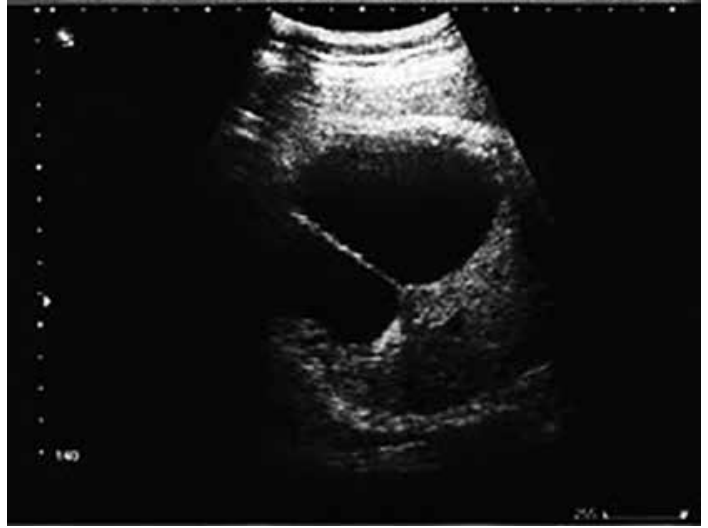

Figure 1. Ultrasound revealed a well-defined, echofree cystic mass measuring $8 \mathrm{~cm} \times 10 \mathrm{~cm}$ on the right side of her uterus with partial septations

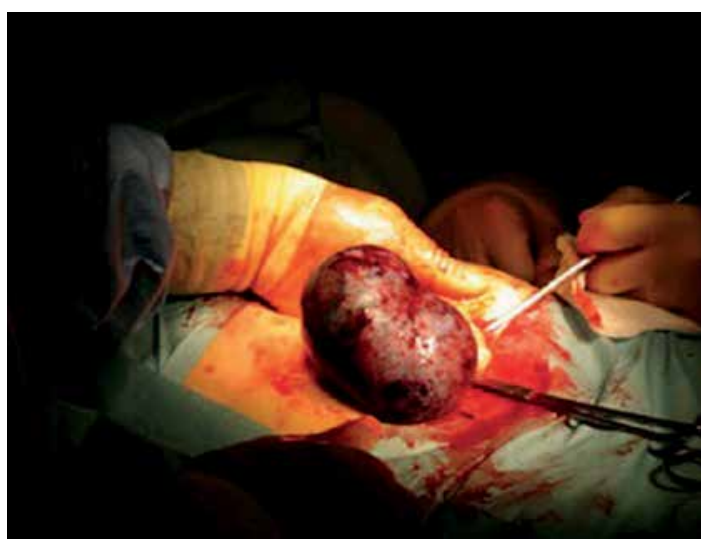

Figure 2. Torted necrotic ovarian mass found at laparotomy

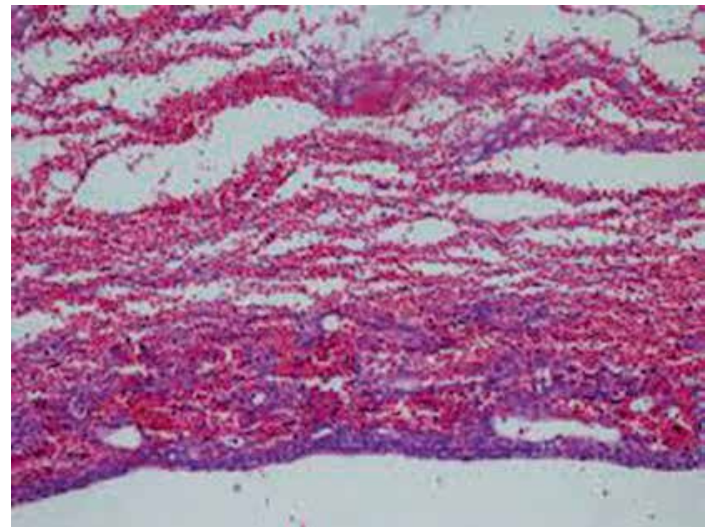

Figure 3. No pathologic diagnosis was made owing to the complete necrosis of the surgical specimen, but there was no evidence of neoplastic proliferation

require salpingo-oophorectomy. Salpingo-oophorectomy is also reasonable for postmenopausal women. In a review study on 135 cases of adnexal torsion, the incidence of malignancy was estimated at $15 \%$, which increased to $25 \%$ in women older than 60 years [10]. According to the findings 
of the present study, given the possibility of neoplastic changes in post-menopausal patients with OT, frozen section technique should be available; if not possible, clinical and imaging procedures can be helpful. However, surgeons must note that extensive necrotic tissues in adnexal masses may result in the misdiagnosis of malignancy in frozen section technique. Therefore, optimal treatment can be achieved via surgical staging in suspicious macroscopic cases. The risk of thromboembolic events should be considered in OT cases. However, the incidence of pulmonary embolism in cases of adnexal torsion is the same before and after untwisting [11].

For most premenopausal patients with ovarian torsion, we recommend detorsion and ovarian conservation rather than salpingo-oophorectomy, even in the case of a darkened, congested ovary. Ovarian cystectomy is often performed if a benign mass is present. Patients with an obviously necrotic ovary or an ovarian mass that is suspicious for malignancy require salpingo-oophorectomy. Salpingo-oophorectomy is also reasonable for postmenopausal women.

\section{Conflict of interest}

The authors declare no conflict of interest.

\section{References}

1. McWilliams GD, Hill MJ, Dietrich CS 3rd. Gynecologic emergencies. Surg Clin North Am 2008; 88: 265-83.

2. Huchon C, Fauconnier A. Adnexal torsion: a literature review. Eur J Obstet Gynecol Reprod Biol 2010; 150: 8-12.

3. Liberis V, Tsikouras P, Zografos C, et al. The contribution of laparoscopy to the diagnosis of adnexal masses in young and premenopausal women. Eur J Gynaecol Oncol 2009; 30: 402-7.

4. Huang TY, Lau BH, Lin LW, Wang TL, Chong CF, Chen CC. Ovarian cyst torsion in a toddler. Am J Emerg Med 2009; 27: 632.e1-3.e1.

5. Hibbard LT. Adnexal torsion. Am J Obstet Gynecol 1985; 152: 456-61.

6. Koonings PP, Grimes DA. Adnexal torsion in postmenopausal women. Obstet Gynecol 1989; 73: 11-2.

7. Alcázar JL, Royo P, Jurado M, et al. Triage for surgical management of ovarian tumors in asymptomatic women: assessment of an ultrasound-based scoring system. Ultrasound Obstet Gynecol 2008; 32: 220-5.

8. Canis M, Jardon K, Boulleret C, et al. Management of adnexal tumors: role and risks of laparoscopy. Gynecol Obstet Fertil 2001; 29: 278-87.

9. Sasaki KJ, Miller CE. Adnexal torsion: review of the literature. J Minim Invasive Gynecol 2014; 21: 196-202.

10. Ryan MF, Desai BK. Ovarian torsion in a 5 -year old: a case report and review. Case Rep Emerg Med 2012; 2012: 679121.

11. Lee JW, Kim CJ, Lee JE, et al. Selected adnexal cystic masses in postmenopausal women can be safely managed by laparoscopy. J Korean Med Sci 2005; 20: 468-72. 